\title{
Apparent Jet Speed versus Optical Classification: An Effective Test of Unified Model in Powerful Radio Sources
}

\author{
${ }^{1}$ J.N. Ogwo, ${ }^{2}$ A.C. Alago \\ ${ }^{1,2}$ Department of Physics, Abia State University Uturu, Abia State.
}

\begin{abstract}
We have carried out some statistical tests of relativistic beaming and radio source orientation scenarios using optical class, apparent jet speed $\left(\beta_{a p p}\right)$ and redshift $(1+z)$ of a sample from Kellermann et al (2004) as orientation indicators. The final compilation consists of 96 sources. 11 FRII radio galaxies and 85 radio-loud quasars were used for the analyses. Our results show that quasars have higher apparent jet speed than galaxies with the respective mean values as 6.833 and 1.209. This result is consistent with the simple relativistic beaming and orientation-based unification hypotheses in which quasars are the beamed counterparts of powerful radio galaxies which form the isotropic parent population. In order to further test the unification scheme for these objects, correlation analysis of the observed parameters for the two optical classes was performed. The result shows strong evidence that FRII radio galaxies and quasars are intrinsically the same but appear different due to relativistic beaming and orientation effects.
\end{abstract}

\section{Introduction}

Many properties of active galactic nuclei (AGN) such as superluminal motion can be readily understood in terms of the relativistic beaming and orientation based models in which the radio emission from the core of these objects is highly anisotropic and their extended emission is isotropic. These models have become the bedrock of the popular unification paradigms for radio-loud quasars and FRII radio galaxies in which radio-loud quasars are seen within a small angle to the line of sight while FRII radio galaxies are their isotropic parent population. This is so because, in the orientation-based unified scheme for radio galaxies and quasars, these objects are believed to be intrinsically similar but appear to be different because of their different orientations to the line of sight. There has been a reasonable degree of evidence in different wave bands in support of such a scheme (Barthel 1998; Urry \& Padovani 1995). High luminosity radio sources which include radio-loud quasars and FRII radio galaxies have total radio luminosity (in the blue band) $\mathrm{P} \geq 10^{42}$ ergs- $^{1}$ with more highly ionized accretion disk (Wu et al 2002). In relativistic beaming, charged particles moving close to the velocity of light will emit electromagnetic radiation in a narrow beam in the direction of motion. The width of the beam so produced varies inversely with the speed of the particles. Beaming is common in many AGN galaxies. Relativistic beaming is of importance for a jet which is oriented close to the line of sight from the AGN to Earth. At very small angles to the line of sight of the observer, beaming effects become large and the observed luminosity of a jet becomes higher than the intrinsic luminosity. The jet on the other side, moving away from the earth (counter jet) has less luminosity than its intrinsic luminosity. In a very simple version, the relativistic beaming model postulates that the radio emission from a jet moving at small angles to the line of sight would be Doppler boosted. Hence a jet moving with relativistic speed at angle $\square$ to the observer's line of sight would have its observed flux density given by (Kollgaard 1994).

where

$$
\mathrm{S}_{0}(\mathrm{v})=\mathrm{S}_{\mathrm{e}}(\mathrm{v}) \delta^{\mathrm{P}}
$$

$\mathrm{S}_{0}(\mathrm{U})$ is the observed flux density, $\mathrm{S}_{\mathrm{e}}(\mathrm{U})$ is the emitted flux density, $\delta$ is the beaming enhancement factor or Doppler factor and $\mathrm{P}$ is the beaming index. The Doppler factor is an observational factor which depends on the speed of the moving jet and its inclination angle $\square$ to the observer's line of sight and it is expressed (Ubachukwu \& Chukwude 2002) as:

$$
\delta=\gamma^{-1}(1-\beta \cos \square)^{-1}
$$

where $\gamma$ is the Lorentz factor of the moving jet and $\beta$ is the velocity of the jet in units of the speed of light. The beaming index $\mathrm{p}$ in equation (1) is given as

$$
\mathrm{P}=\mathrm{n}+\alpha
$$

Where $\alpha$ is the core spectral index and $\mathrm{n}$ is a parameter which depends on the assumed flow model ( $\mathrm{n}=2$ for continuous beam model and $\mathrm{n}=3$ if the radiating plasma consists of blobs).

In relativistic beaming model, the beam does not change the power law nature of the spectrum but, however its effect can greatly alter the appearances of two distant galaxies which are identical except for different angles to 
the line of sight (Ubachukwu \& Chukwude 2002). Relativistic Doppler boosting in addition to increasing the brightness of a source can also create an illusion that the apparent transverse velocity of a jet exceeds the speed of light

$(\mathrm{v} / \mathrm{c}>1)$

The illusion is called superluminal motion (Gabuzda et al 1994). The simplest orientation based unification scheme shows that the apparent jet speed $\left(\beta_{\text {app }}\right.$ ) of a given radio source depends on the viewing angle as given by

where

$$
\beta_{\text {app }}=\beta \frac{\sin \square}{1-\beta \cos \square}
$$

$\beta_{\text {app }}$ is the observed apparent transverse velocity of the source, $\beta$ is the real transverse velocity and $\square$ is the inclination angle of the source to the observer's line of sight.

\section{Analyses and Results}

The present analyses were carried out using the compilation of Kellermann et el 2004. The sample consists of 96 sources: 11 FRII radio galaxies and 85 radio - loud quasars which provided a complete information about the optical class, redshift as distance indicator and apparent jet speed in units of the speed of light. The tests are aimed at understanding the behavior of the two classes of objects at relatively near physical conditions. That is to determine if there is a continuity in the properties of the two classes, which indicates that any difference could be due to orientation effects. The distributions of the observed parameters for the two classes of objects over a range of frequencies $(15 \mathrm{GHz})$ are compared. Hence analysis of the data for the two classes of objects at exactly the same frequency would ensure that there is no discrepancy in the analyses. The distributions of the observed parameters of the FRII radio galaxies and the radio-loud quasars objects are shown below.

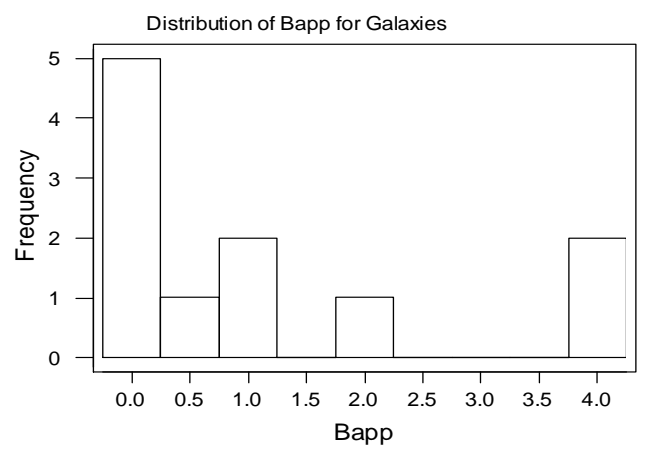

From the above distribution (tig. la) we noticed that galaxies have small range value of apparent jet speed with most of the sources clustered between 0 to 4.0 , in a certain frequency range.

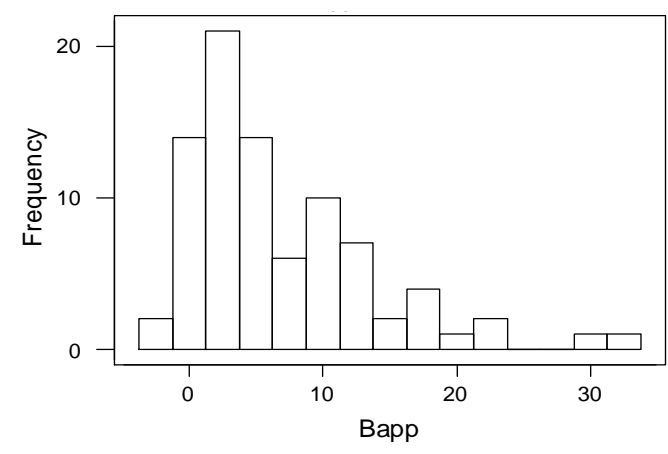

Fig. 1(b): Distribution of $\beta_{\text {app }}$ for Quasars

From fig.1(b): It is observed that for a certain frequency range quasars clustered between 0 to 30 which shows high values of apparent jet speed. 


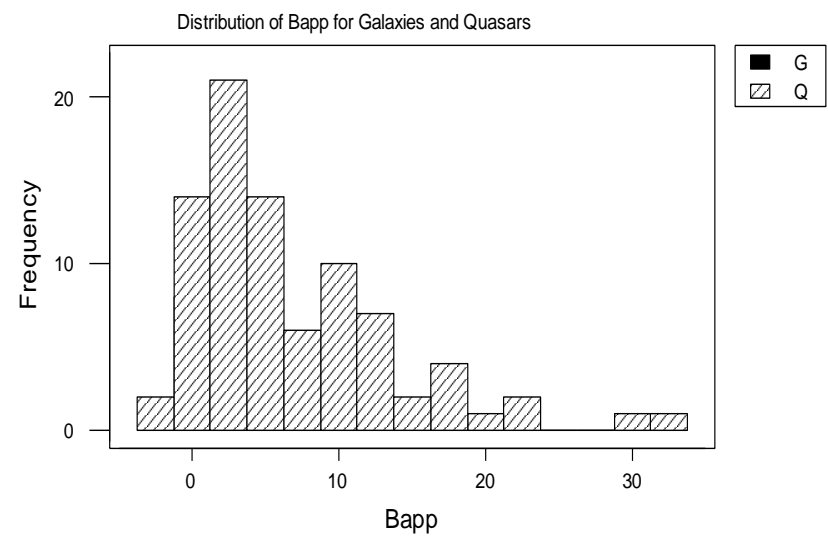

Fig. 1@: Distribution of $\beta_{\text {app }}$ for FRII galaxies and quasars. (Darkened and line filled blocks represents radio galaxies and quasars respectively).

Comparing the distribution for radio galaxies and quasars, we found out that it is dominated with quasars ranging from 0 to 30 while galaxies have no bar representing it. This means, looking at the two objects apparently, they are distinguishable with quasars exhibiting high apparent jet speed than galaxies.

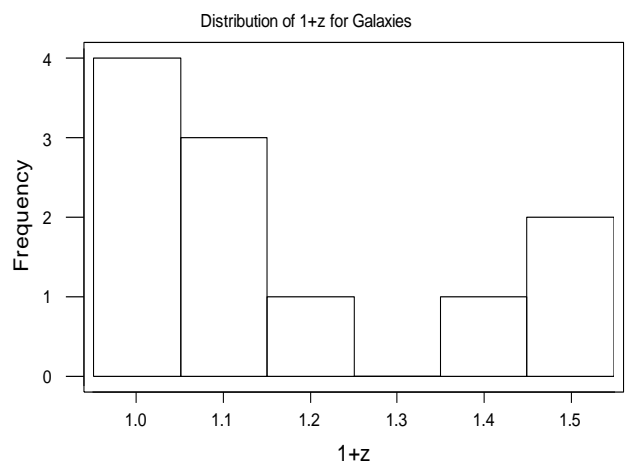

Fig. 2(a): Distribution of $1+\mathrm{z}$ for Galaxies

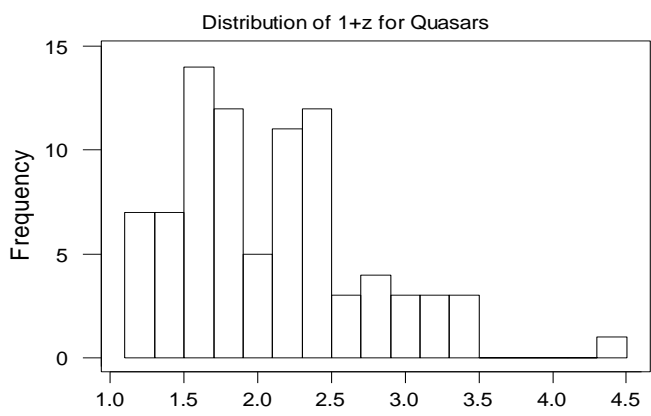

Fig. 2(b): Distribution of $1^{1+z}$ for Quasars 


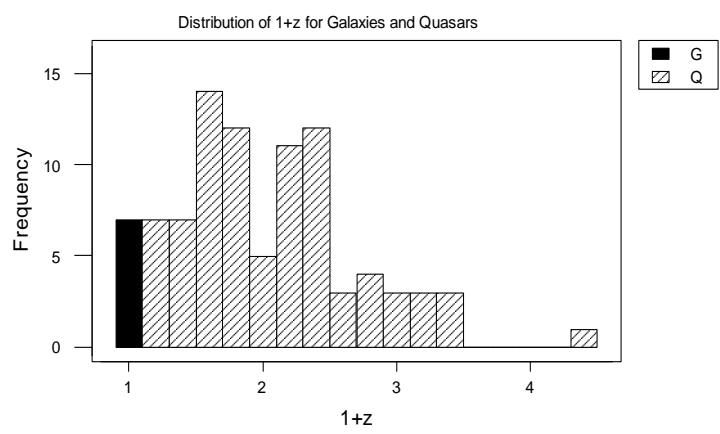

Fig. 2(c) Distribution of $1+z$ for FR rkened

Fig. 2c): Distribution of $1+\mathrm{z}$ for Quasars and FR11 radio galaxies.

Darkened and line filled blocks represents radio galaxies and quasars respectively).

The distributions of redshift (1+z) for FR II radio galaxies and quasars are shown in figures $2 a, 2 b$ and 2c. From figure $2 \mathrm{c}$ it is observed that FRII galaxies are represented by only one bar clustering between 0 to 1 while quasars clustered from one to above four, meaning that FRII galaxies have very low redshift compared to quasars. The study of the correlations among various radio sources parameters has provided very valuable information not only in the understanding of the physics of radio source evolution but has tremendous implications for the relativistic beaming and radio source unification schemes (Saikia et al, 1985), The results of the analysis obtained in this section provide reasonable evidence in support of the FRII- Radio Loud- quasars unified scheme. The degree of dependence between any two parameters is further quantified statistically using the correlation coefficient ( $r$ ). The correlation coefficient could be positive or negative depending on the nature relativistic beaming and orientation- based unified model for the radio loud AGNs of the association $(-1 \leq r \leq 1)$. apparent jet speed $\left(\beta_{\text {app }}\right)$ and redshift $(1+\mathrm{z})$ are good statistical tools for testing the relativistic beaming and orientation- based unified model for the radio loud AGNs. To determine the correlations between some observed parameters of the FRII galaxies and quasars, we show some of the functional relationships in the plots below

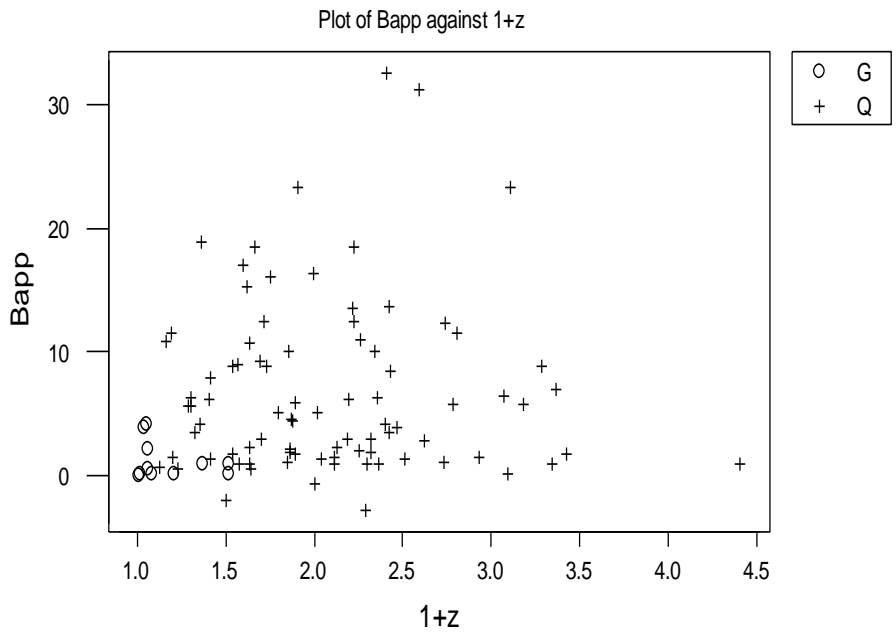

Fig. 3: Plot of the apparent jet speed ( $\left.\beta_{\text {app }}\right)$ against the source redshift $(1+\mathrm{z})$. Circles and pluses represent galaxies and quasars respectively.

From the correlation analysis, it shows that apparent jet speed and redshift of FRII galaxies and quasars are anti-correlated with correlation coefficient ( $\mathrm{r}$ ) of -0.281 and 0.002 respectively. This anti- correlation is expected to provide a qualitative test of the beaming scenarios. 


\section{Discussion of Results}

The distributions of radio sources parameters were used to carry out a quantitative investigation of the relativistic beaming and radio source orientation based FRII galaxies-quasars unified scheme. A direct comparison of the distributions of the observed parameters of the two different classes of radio sources in figures (1a) and (1b) reveals apparent continuity of the properties of the two classes of radio sources.

Kapahi (1998) stated that if radio sources are assumed to be randomly distributed in space the mean and median values would be the best for characterizing these source parameters. Figures (1a) and (1b) show the distributions of $\beta_{\text {app }}$ for galaxies and quasars respectively. A look at the distributions for these objects shows that they are clearly distinguishable. FRII galaxies have a mean value of $1.209 \pm 1.523$ and median value of $0.500 \pm 1.523$. On the other hand, the mean and median values for quasars are (6.833 and 5.100) respectively. The same distributions reveal the minimum and maximum values of $\beta_{\text {app }}$ for FRII galaxies as 0.040 and 4.200 while those of quasars are -2.80 and 32.500 respectively. The major observed difference in the distribution of the apparent jet speed for FRII galaxies and quasars is that quasars shows higher mean value of apparent speed than galaxies; which is in accordance to Barthel et al (1989), which states that FRII radio galaxies form the unbeamed parent population of quasars that leads to a higher value of orientation angle but a lower value of apparent speed. The observe larger apparent speed for quasars could be attributed to Doppler Effect or high degree of superluminal motion (Orr \& Browne 1982).

The redshift is believed to be a statistical indicator of the relativistic beaming scenario. The mean and median values of redshift $(1+\mathrm{z})$ for FRII radio galaxies are 1.1727 and 1.0570 respectively compared with 2.0844 and 1.999 for quasar.

\section{Conclusion}

In this paper the observed parameters of the two classes of powerful radio sources (FRII radio galaxies and quasars) apparent speed and redshift have been used as beaming and radio source orientation indicators. Distributive statistics and linear correlation were used in the analyses. The results from these analyses seem to support the beaming and orientation hypothesis which states that the two classes of objects are intrinsically similar but appear to be different because of their different orientations to the line of sight. The quasars are oriented close to the line of sight whereas the radio galaxies lie close to the plane of the sky.

[1] Barthel, P. D., 1989, Astrophysical Journal, 366, 606

\section{References}

[2] Gabuzda, D. C., Mullan, C. M., Cawthorne, T. V., Wardle, J. F. C., and Roberts, D. H., (1994), Astrophysical journal, 435, 140 .

[3] Kapahi, V. k. (1987). The effect of Luminosity-Redshift correlation on the Analysis of the Angular Size-Redshift Relation for Radio Galaxies. IAU Symp 124: P. 125.

[4] Kellerman, K. I., Lister, M. 1. and Homan, D. C. (2004). Sub-Nuclei III-Kinematics ofparsel-scale Radio Jets. The Astrophysical Journal 609: 539-563

[5] Kollgaard, R. I. (1994). Unified Schemes for Radio-Loud AGN. Vistas in Astronomy38:29.

[6] Orr, M. J., Browne I. W. A. (1982). A constraint on the Standard Beaming Model forSuperluminal Sources. Mon. Not R. Astron Soc. 200:1067.

[7] Scheuer, P.A.G. 1987 in Superluminal Radio. Sources eds. J.A. Zensus and T.J. Pearson (Cambridge Univ. Press) P. 104.

[8] Smith, F. G., Heckman, T. M. (1986). Radio Observations of jets. Larges Scales Astrophysical Journal 368:611.

[9] Ubachukwu, A. A. (1998). A Constraint on the Standard Beaming Model for Superluminal Sources. Astrophysics and Space Science 257:23.

[10] Ubachukwu, A. A., Chukwude A. E. (2002). Asymmetries in Powerful Radio Source;Journal for Astrophysics and Astronomy 23:253.

[11] Urry, C. M., Padovani P. (1995). Unified Schemes for RadioLoud AGN. Publications of the Astronomical Society of the Pacific 107:803-845. 